\title{
Optimized Scratch Drive Actuator for Tethered Nanometer Positioning of Chip-Sized Components
}

\author{
Ryan J. Linderman, Victor M. Bright \\ NSF Center for Advanced Manufacturing and Packaging of Microwave, \\ Optical, and Digital Electronics (http://mems.colorado.edu) \\ University of Colorado at Boulder, CO 80309-0427
}

\begin{abstract}
A novel surface micromachined robot, capable of pushing a $2 \times 2 \times 0.5 \mathrm{~mm}$ chip several millimeters while being powered by a gold wire tether is presented. The micro robot uses a unique assembly and packaging process and is created from large arrays of scratch drive actuators (SDA) tethered by gold wires bonded prior to release in an HF acid bath. The wire tether allows for robot transfer to other substrates and driving surfaces, where assembly line positioning tasks can be performed. A device model has been derived to define optimum SDA plate design for any fabrication process. The driving signal has also been optimized, consuming much less electrical power than previously published signals. Because the step size of the individual SDA is approximately 30 nanometers, the tethered SDA robots are expected to achieve similar placement precision for chip sized components. The SDA robot (Scratchuator) demonstrates the potential for low cost nanometer positioning of chip sized components for alignment and flip chip assembly.
\end{abstract}

\section{INTRODUCTION}

The scratch drive actuator is the only micro system to provide high precision $(\sim 30 \mathrm{~nm})$ long range motion with a large output force. Previously published papers have highlighted the use of the SDA for optical autocorrelators[1], 3D micro stages[2] as well as novel mechanisms for ultra-thin active catheters[3]. The stepping motion of the SDA occurs when an electrostatic load deflects the plate until the bushing is scratched forward upon which the voltage is reduced and the plate is pulled forward (see figure 3 ). Little modeling of optimum plate design and voltage response has been published on SDA technology. Previously published SDA papers have reported plate designs for a specific fabrication process, however when an alternative fabrication method is used, the SDA plate must be redesigned to account for the new bushing geometry, plate thickness, insulator dielectric properties, Young's modulus, etc. (see figure 1). In this paper modeling functions to define optimum plate length for any fabrication process and material selection are presented to assist in the development of new applications and alternative microsystem material choices.

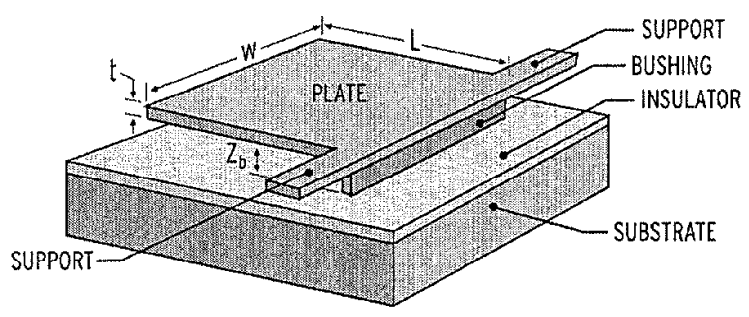

Figure 1. Scratch drive actuator nomenclature.

Once the individual SDA has been modeled for a given fabrication process, an optimum plate design can be chosen and experimentally verified. By designing a series of test stands with incremental variations in design parameters, the experimental optimum can be evaluated with intended applications in mind.
Because the SDA offers high force, long range and nanometer precision placement capabilities, it is well suited to meet the needs of future optics and flip-chip assembly operations. The limits of precision machined lead screws and large scale positioning systems have already been realized at significant costs. Thus the development of a microsystem based alignment and positioning actuator, such as shown in figure 2 , stands to have a dramatic impact on chip and wafer based assembly operations.

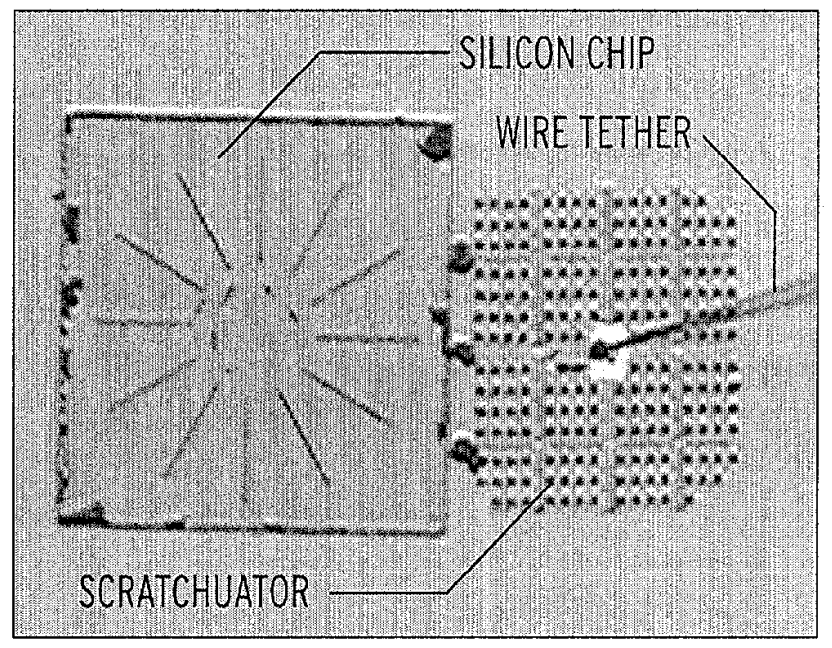

Figure 2. 188 SDA robot (Scratchuator) positioning a $2 \times 2 \times 0.5 \mathrm{~mm}$ chip.

\section{DEVICE MODEL}

The problem encountered when designing SDA for a given fabrication process is defining an appropriate plate length for a given stiffness material and bushing height. Plates that are too long flatten out against the insulating nitride layer after the snap through voltage is reached - limiting the output force of the SDA. Plates that are too short require exceptionally high voltage levels to step forward which have adverse effects on wear and life span of the mechanism. Because nearly all the work from the SDA is produced from longitudinal deflection, the width of the plate does not play a dominant role in optimization however, the width does have a dramatic effect on the squeeze film damping and frequency response of the SDA.

Two characteristic deflections must be modeled in order to determine the optimum plate length for a given fabrication process. The snap through voltage occurs when the electrostatic loading critically overpowers the elastic stiffness of the SDA resulting in the plate tip snapping down to touch the nitride dielectric layer. The priming deflection is the next function of plate length to be modeled and occurs when the SDA plate tip is deflected enough to flatten to a zero slope at the free end as shown in figure 3. For an optimized SDA plate, the snap-through voltage is equal to the priming voltage - resulting in the best balance of drag friction and voltage response. 

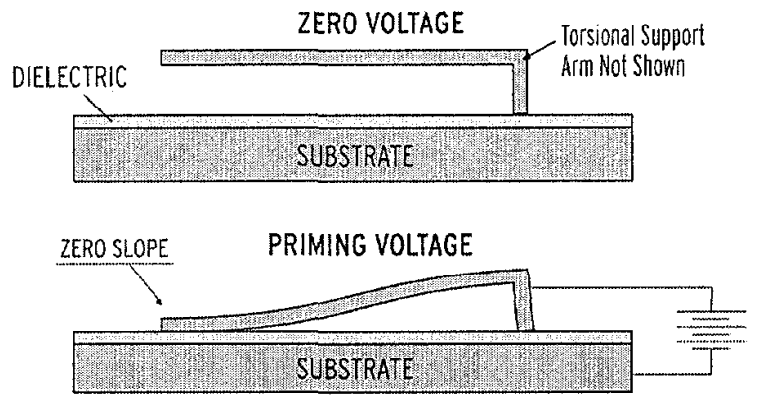

STEPPING VOLTAGE

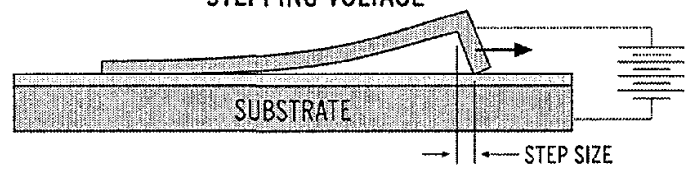

Figure 3. Concept of SDA stepping motion. In order to produce forward motion the SDA oscillates between the stepping and priming deflection.
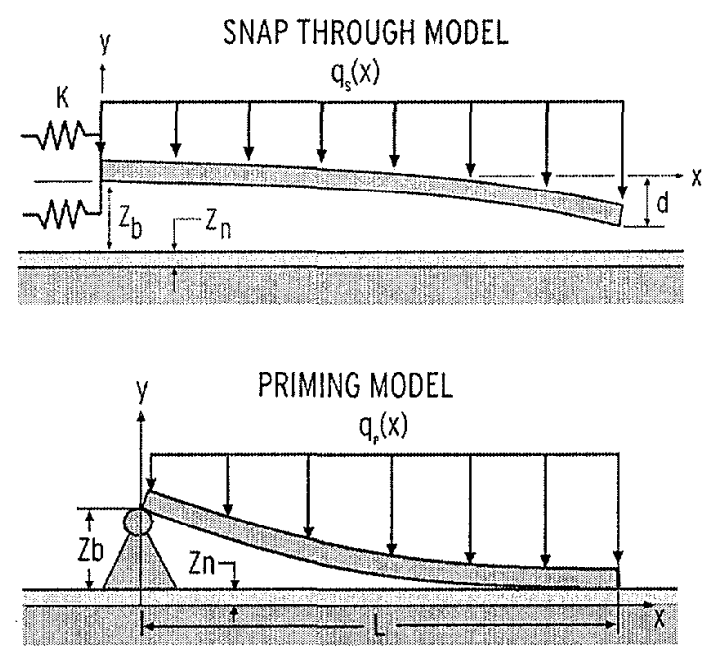

Figure 4. Free body diagrams for snap through and priming model.

The relationship for tip deflection of a torsionally supported cantilever with a distributed electrostatic load, $q_{s}(x)$, is given by[4]:

$$
d=\int_{0}^{L} q_{S}(x) \frac{w x}{6 E I}\left(3 x L-x^{2}+6 K L\right) d x
$$

Where $K$ is the torsional flexibility defined by the rectangular support arm dimensions, $\varepsilon$ is the permittivity of free space, $E$ is Young's modulus, and $I$ is the moment of inertia. Assuming square law curvature for the electrostatic loading[5] and a maximum separation distance of $Z m=Z b+Z n$ :

$$
q_{s}(x)=\frac{\varepsilon \kappa_{1}}{2}\left[\frac{V}{z_{\text {in }}-\left(\frac{x}{L}\right)^{2} d}\right]^{2}
$$

Where $V$ is the applied voltage, $\kappa_{1}$ is the effective dielectric constant of the air $\left(\kappa_{\mathrm{A}}-1\right)$ and nitride $\left(\kappa_{\mathrm{N}}-5.7\right)$ layers below the SDA plate for the snap through model. Because the plate is curved towards the nitride layer, $\kappa_{1}$ is estimated based on the average height of the plate. For the MUMPs process and a bushing of 1.5 $\mu \mathrm{m}$, a valuc for $\kappa_{1}$ of 2.0 works wcll for the model presented. Substituting equation (2) into (1), integrating and solving for the voltage as a function of deflection yields equation (3).

$$
V=\frac{E I}{W E \kappa_{1} L^{2}} \frac{\sqrt{\frac{6 w E K_{1}}{d E I}\left[2 d \frac{Z_{m}+3 \frac{K d}{L}}{Z_{m}\left(Z_{m}-d\right)}-\frac{3 \sqrt{d}}{\sqrt{Z_{m}}} \tanh ^{-1}\left(\frac{d}{\sqrt{Z_{m} d}}\right)-\ln \left(\frac{Z_{m}-d}{Z_{m}}\right)\right]}}{\left(\frac{3 K}{Z_{m} L}\right) \frac{1}{Z_{m}-d}-\frac{3}{2 d \sqrt{Z_{m} d}} \tanh ^{-1}\left(\frac{d}{\sqrt{Z_{m} d}}\right)-\frac{1}{2 d^{2}} \ln \left(\frac{Z_{m}-d}{Z_{m}}\right)}
$$

Equation (3) can then be differentiated with respect to $d$ to determine the deflection at snap through given by:

$$
d=\frac{45}{8} Z_{m} \frac{L+4 K}{13 L+45 K}
$$

Substituting (4) back into (3) defines the snap through voltage as a function of SDA plate length. The next step is to derive an expression for the priming voltage as a function of SDA plate length.

Based on the geometry of the priming model the slope at the bushing, $\mathrm{Zb} / \mathrm{L}$, can be substituted into a standard formula for simply supported beams[4].

$$
\frac{Z_{b}}{L}=\frac{1}{6 L E I} \int_{0}^{L} q_{p}(x)(L-x)\left[L^{2}-(L-x)^{2}\right] d x
$$

The electrostatic loading function for the priming model differs from the snap through loading due to the fact that the plate has collapsed against the insulating layer.

$$
q_{p}(x)=\frac{\varepsilon \kappa_{2}}{2}\left[\frac{V}{Z_{n}+\left(\frac{x}{L}\right)^{2} Z_{b}}\right]^{2}
$$

Where $\kappa_{2}$ is the effective dielectric for the nitride and air layers as defined by the priming model. The value for $\kappa_{2}$ is larger than $\kappa_{1}$ because the effective dielectric for the priming model is predominantly the value for silicon nitride. A value of 2.7 for $\kappa_{2}$ is a reasonable estimate for a MUMPS SDA with primed deflection. Substituting $\mathrm{q}_{\mathrm{p}}(\mathrm{x})$, into (5), integrating and solving for the voltage yields.

$$
V_{p}=\sqrt{\frac{24 Z_{b}^{2} E I}{\varepsilon K_{2} L^{4} w}\left(\frac{3}{\sqrt{Z_{n} Z_{b}}} \tan ^{-1}\left(\frac{Z_{b}}{\sqrt{Z_{b} Z_{n}}}\right)-\frac{\ln \left(Z_{n}+Z_{b}\right)}{Z_{b}}-\frac{2}{Z_{n}}+\ln Z_{n}\right)^{-1}}
$$

With functions for both the snap through and priming voltage derived, an optimum plate is defined by plotting both functions verses plate length. Figure 5 plots equation (7) and the result of substituting (4) into (3) for the CRONOS $^{(1)}$ MUMPs fabrication data listed in table 1 .

\section{CRONOS $^{\circledast}$ MUMPs Fabricated SDA Data}

$\begin{array}{ll}\text { Poly-2 thickness (plate, supports) } & 1.5 \mu \mathrm{m} \\ \text { Bushing (dimple + via) } & 1.5 \mu \mathrm{m} \\ \text { Support length } & 29 \mu \mathrm{m} \\ \text { Support width } & 3 \mu \mathrm{m} \\ \text { Nitride layer thickness } & 0.6 \mu \mathrm{m} \\ \text { Nitride dielectric constant } & 5.7 \\ \text { Young's modulus (polysilicon) } & 169 \mathrm{GPa} \\ \text { SDA width } & 65 \mu \mathrm{m}\end{array}$

Table 1. SDA model data for CRONOS ${ }^{\circledR}$ MUPs fabrication. 
VOLTAGE RESPONSE VS. SDA PLATE LENGTH

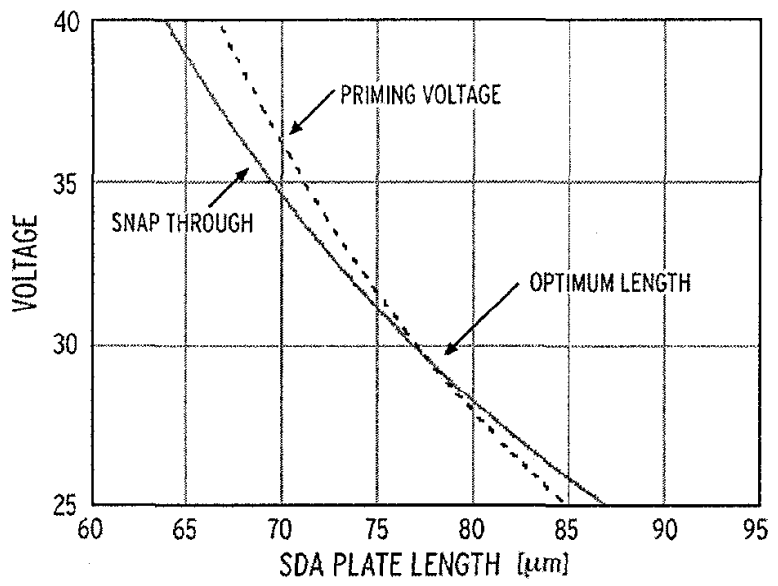

Figure 5. Optimum SDA plate length modeling results.

It should be noted from figure 5 that small errors in either voltage function can have a large effect on the predicted optimum length. Thus, the model should be used to provide an estimate for an initial starting point for experimental verification.

Because the priming deflection is held between stepping cycles, the optimal drive signal for SDAs is an AC signal offset by a DC priming voltage. This drive signal consumes significantly less electrical power than previously published drive signals[1],[3] and results in extended fatigue life and reduced wear to SDA mechanisms. Techniques for experimental evaluation are considered next.

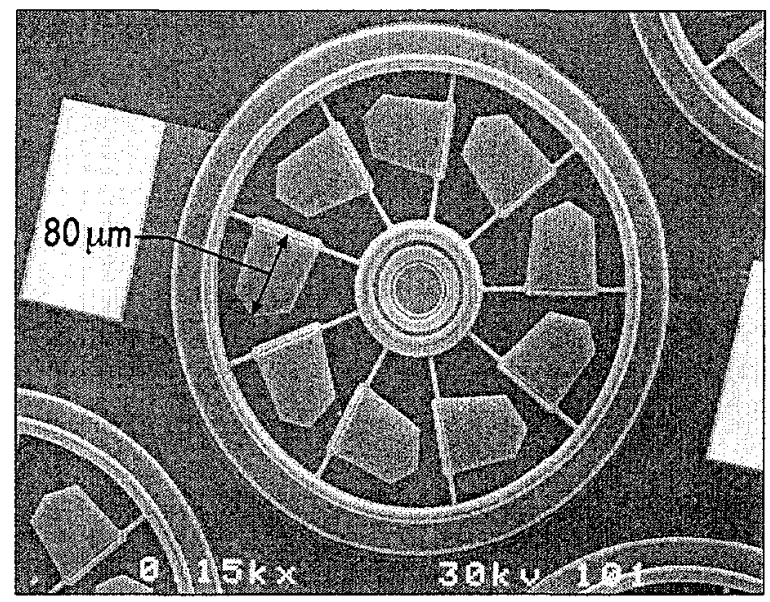

Figure 6. Rotary SDA test stand.

\section{EXPERIMENTAL VERIFICATION}

The model was verified using a series of rotary SDA test stands (see figure 6) with incremental changes in plate length to characterize minimum driving voltage with respect to plate length. The rotary motors were fabricated using the CRONOS ${ }^{\circledR}$ MUMPs process corresponding to Table 1, with plate lengths varying from 65 to $80 \mu \mathrm{m}$ in $5 \mu \mathrm{m}$ increments. Based on the modeling results, the driving voltage will be minimized at the experimental optimum due to the balancing of drag and friction forces. The experimental results are plotted in figure 7 with the error bars representing the average standard deviation for eight device data points at each plate length. The error is largely due to variations in the release process and storage conditions of individual devices.
Minimum Driving Voltage vs. Plate Length

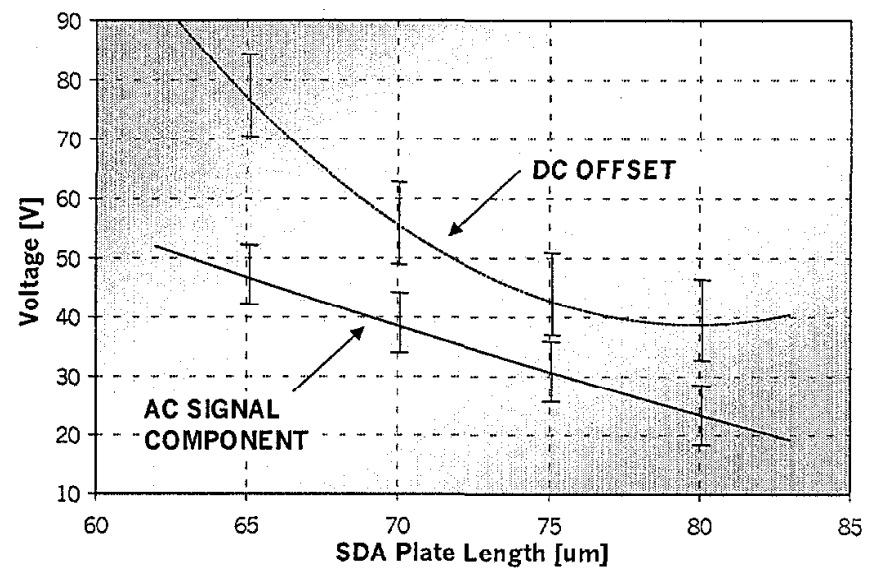

Figure 7. Experimental minimum driving voltage for a $1 \mathrm{kHz}$ signal.

The AC component used to drive the SDAs shown in figure 7 was recorded as a zero to peak value with all tests using a $1 \mathrm{kHz}$ signal. It should be noted that the drive signal voltages reach a minimum for a plate length of approximately $78 \mu \mathrm{m}$, which agrees well with the modeling prediction for optimum length (see figure 5). Other tests have been performed using linear arrays to determine the output force from SDA arrays as a function of plate length. These tests have revealed a nearly linear decrease in output force from 20 to $9 \mu \mathrm{N}$ for plate lengths ranging from 55 to $85 \mu \mathrm{m}$. Thus for short range and high force SDA applications a shorter plate length than the predictcd optimum may bc requircd. However, as can be observed from figure 7 , the increase in output force from shorter plate lengths comes at a cost of higher drive voltages, and in turn reduced fatigue life due to the adverse wear affects of the higher voltages.

\section{TETHERED SDA ROBOTS}

With the design of the SDA optimized, the actuator can be arrayed in large numbers to provide forces capable of moving chip sized and larger components. The SDA robot (Scratchuator) shown in figure 8 was made from an array of 188 SDA and is tethered by a $25 \mu \mathrm{m}$ gold wire. The gold wire is bonded to the Scratchuator before release in an HF bath which separates the device from the substrate it was fabricated on. The gold bumps on the front end of the Scratchuator are used as a bumper for pushing objects or as a means to bond the device to a larger ceramic stage

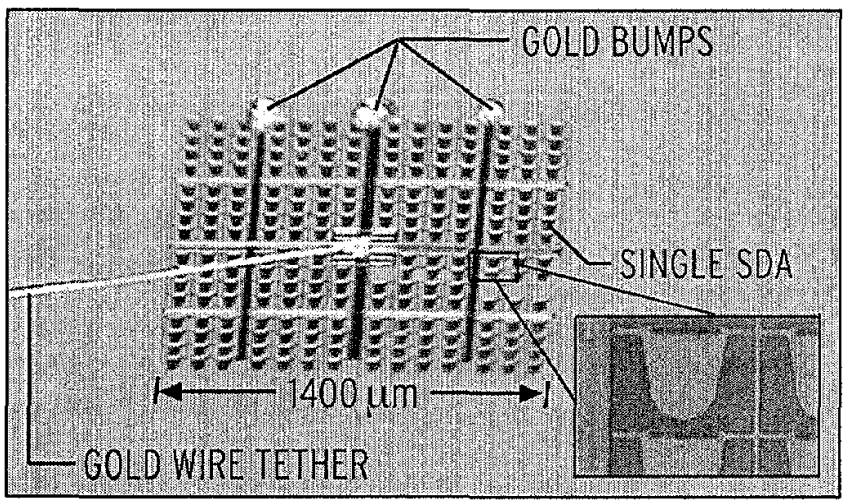

Figure 8. Wire tethered Scratchuator with array of 188 SDA. 


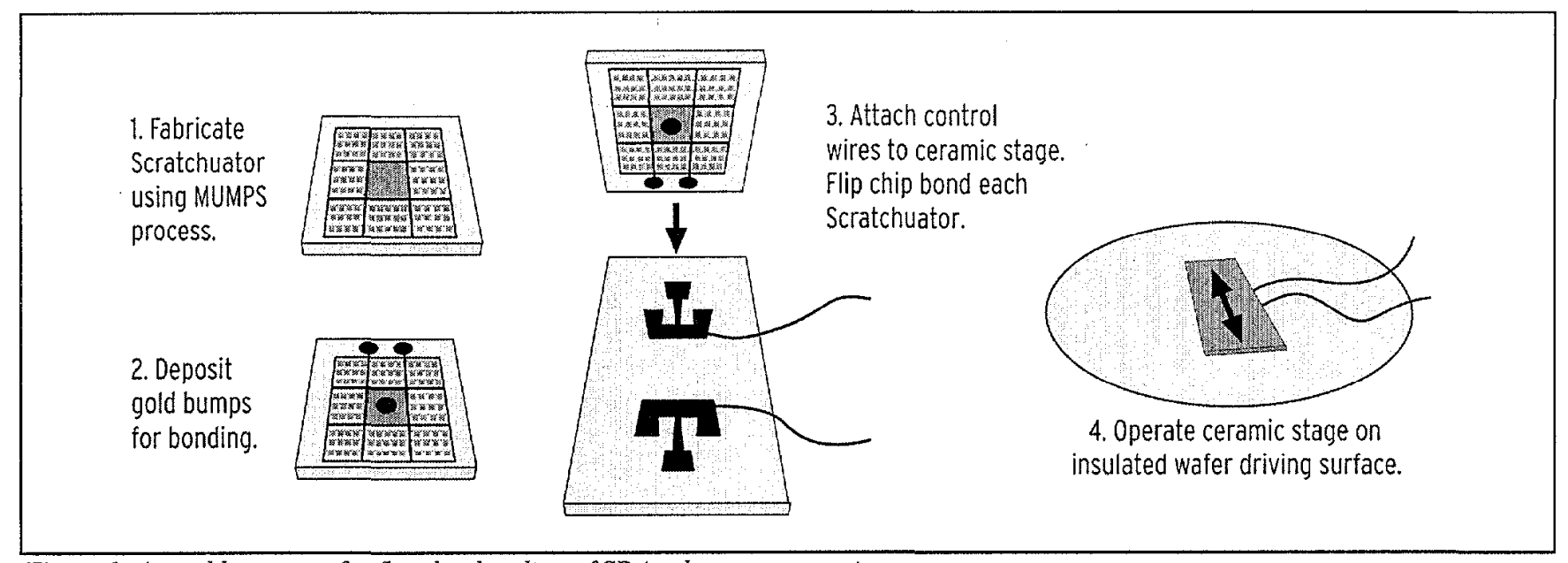

Figure 9. Assembly process for flip chip bonding of SDA robots to a ceramic stage.

as shown in figure 10. An SDA array is required for each desired direction of motion when positioning objects. Assembly techniques for the ceramic stage are shown in figure 9.

Because Scratchuators can be attached uniformly to the bottom of a ceramic substrate, large forces and multiple directions of motion are expected from a level stage designed to withstand the normal forces required for use as a flip chip bonding aligner. An silicon wafer can be used as a large driving surface for the Scratchuators as it has a smooth uniform surface with a ground plane and insulating layer.

Preliminary testing has shown a single Scratchuator (figure 8 ) is capable of pushing a $2 \times 2 \times 0.5 \mathrm{~mm}$ silicon chip over 8 millimeters whilc being tethered by a $25 \mu \mathrm{m}$ gold wire even after nearly half the SDAs from the array have been damaged. Failure mechanisms are related to the stiction of individual SDA to the driving surface which causes fracturing when the array is repositioned. Proper designs require a strong central frame that minimizes stress concentrations at the supports of the SDA.

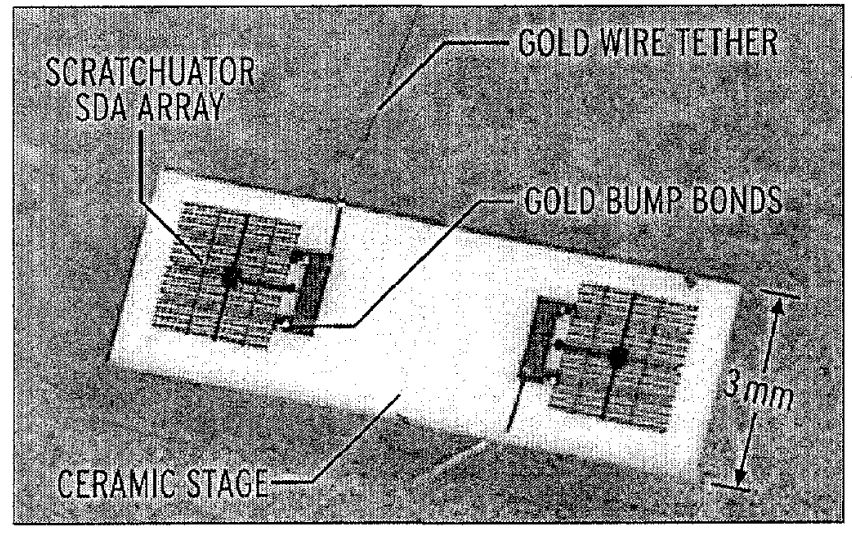

Figure 10. Bottom view of ceramic stage with two Scratchuators gold bump bonded. The gold wire tethers provide electrical power to each Scratchuator allowing controlled motion in two directions when driven across an insulated wafer surface.

\section{CONCLUSIONS}

A novel surface micromachined robot, capable of pushing a $2 \times 2 \times 0.5 \mathrm{~mm}$ silicon chip over 8 millimeters while being powered by a $25 \mu \mathrm{m}$ gold wire tether has been developed. The micro robot uses wire bonding and flip chip techniques for assembly and is created from large arrays of scratch drive actuators (SDA). A model has been derived to define optimum SDA plate design for any fabrication process. The robot is transferred to the large driving surface of an insulated wafer, where assembly line positioning tasks can be performed. The driving signal consists of an $A C$ stepping component with a DC offset used to hold the SDA in a primed deflection between steps. Because the step size of the SDA is approximately 30 nanometers, similar placement precision is expected for positioning of components. The SDA robot (Scratchuator) demonstrates the potential for low cost nanometer alignment of components for flip chip assembly.

\section{ACKNOWLEDGEMENTS}

This project is supported by the Department of Defense (MDA904-97-C-0320), the Defense Advanced Research Projects Agency (DARPA), the Air Force Research Laboratory, Air Force Materiel Command, USAF, under agreement number F30602-98$1-0219$.

\section{REFERENCES}

1. G. D. Su, L. Y. Lin and M. C. Wu, "Single-chip femtosecond autocorrelator realized by surface-micromachined integrated optics," in Proc. Conf. Lasers and Electro-Optics (CLEO), Baltimore, MD, 1997, paper CWL2.

2. L.Fan, M.C. Wu, K. Choquette, and M.H. Crawford, "Selfassembled microactuated XYZ stages for optical scanning and alignment," in Proc. 1997 Int. Conf. Solid-State Sensors and Actuators (TRANSDUCERS 97), 1997, paper 2A2.01.

3. G. Bourbon, P. Minotti, P. Langlet, T. Masuzawa and H. Fujita, "Three dimensional active microcatheter combining shape memory alloy actuators and direct-drive tubular electrostatic micromotors," SPIE Conference on Micromachined Devices and Components IV, Santa Clara, California, September 1998, SPIE Vol. 3514 pp. 147-158.

4. R. B. Hopkins, "Design analysis of shafts and beams," McGraw-Hill, 1970.

5. K. E. Petersen, "Dynamic micromechanics on silicon: techniques and devices," IEEE Transactions on Electron Devices, Vol ED-25, No. 10 pp. 1241-1250, October 1978. 